\title{
Towards sustainable ICT
}

\author{
Michela $\mathrm{Meo}^{1, *}$ \\ ${ }^{1}$ Politecnico di Torino, Corso Duca degli Abruzzi 24, 10129 Torino, Italy
}

\begin{abstract}
While information and communication technologies (ICT) can play a crucial role in tackling issues related to sustainability, and reducing resource consumption in every sector, they also consume huge amounts of energy, and this, combined with the predicted rapid growth of demand for communication services, is expected to make the whole sector more and more energy-hungry in the coming years. The sustainability of ICT itself is also becoming an urgent challenge. Actions can be taken in several directions: i) introducing a massive and pervasive use of renewable energy sources, a step that requires the design of communication services to be revised; ii) enforcing rules and policies that induce the provisioning of sustainable services and promote the adoption of proper attitudes; and iii) involving the users who will become conscious consumers and participate in crowdsourcing projects to monitor consumption and foster energy-aware behaviours.
\end{abstract}

\section{ICT as a set of enabling technologies}

When tackling the issue of sustainability, and the several challenges associated with it, information and communication technology (ICT) has always been perceived very positively. Indeed, ICT enables the development of innovations and solutions that can reduce the consumption of resources in all sectors.

Several of the proposed solutions are similar in spirit, sharing a general approach and having the use of some techniques in common: sensors or devices to monitor and collect data, a communication infrastructure to carry the data to a control centre, and a central management unit that takes decision about possible actions for the optimisation of the use of resources. We can consider examples from different sectors. In energy monitoring systems for buildings, a sensor network is used to monitor a number of parameters: temperature, humidity, and the presence of people. A control unit operates the cooling and heating systems in an optimal way in terms of electricity consumption. A second example, from a very different sector, is predictive maintenance for power grids. The electricity distribution network is equipped with sensors that collect data and information about the current working conditions of the elements of the grid, as well as the environmental parameters. The data feeds a control unit that learns when critical conditions might arise, and takes actions and countermeasures to reduce the occurrence of failures or attenuate their impact by reacting promptly. Similar examples can be found from almost every sector, from manufacturing systems, to urban mobility, from logistics to energy generation plants. It is estimated that each sector will benefit from ICT and will experience a reduction in energy consumption of up to $30 \%$ [1].
Digitalisation allows the substitution of resource demanding activities with corresponding light e-versions that reduce the need to move goods and people, by moving bits, a much less demanding effort. This is the case in e-learning, teleworking, dematerialisation, ...

The basis of these possibilities is the incredible increase in computing, storage and transmission capacity. The famous Moore's law, first formulated in the 1960s and then updated, states that the number of transistors per chip (i.e., the number of elementary units of information storage and computation that can fit into a small chip) will double every 18 months. We carry today, in the smartphone in our pocket, the computational power that was needed to reach the moon in the late 1960s. Similarly, transmission capacity has grown from the order of the Gbit/s of copper technology to tens of $\mathrm{Tbit} / \mathrm{s}\left(10^{\wedge} 12 \mathrm{bit} / \mathrm{s}\right)$ using optical fibre cable technology.

\section{ICT sustainability}

While ICT plays a positive role in enabling innovations for reductions in resource consumption, some studies have recently estimated that the ICT sector itself consumes huge amounts of electricity and it is responsible for about $3.5 \%$ of global emissions [2].

As reported in 121 all segments of ICT contribute to consumption with a relatively even share. PCs and monitors, and the communication infrastructure, are responsible for about $40 \%$, data storage, computing and management for $23 \%$. It is estimated that in the US alone, data centres (those big aggregations of storage and computing facilities that collect, store and elaborate data, provide contents and cloud services) consume 90 billion $\mathrm{kWh}$ per year, the equivalent of 30 giant power plants of

\footnotetext{
* Corresponding author: michela.meo@polito.it
} 
more than $500 \mathrm{MW}$ each; an amount that corresponds to $3 \%$ of the entire electricity consumption of the country [3].

Communication infrastructure consumption is dominated by the access networks, which bring connectivity in the last hop (the so-called "last mile") to the users. Access networks comprise a very large number of devices that individually consume relatively little, but have a large aggregated total consumption. The consumption of a base station, the fundamental element of the mobile access network, is in the order of $\mathrm{kW}$, but it is estimated that there are seven million sites worldwide, with several base stations per site. For a mobile network operator, the power required for all the base stations can reach $80 \%$ of the operational cost of the whole network.

This data suggests that actions for the reduction of these consumptions are needed in all segments of the ICT sector. Indeed, with the exceptions of cases in which devices rely on small batteries, such as wireless sensors and terminals, usually the design of ICT technologies has not considered energy consumption and supply as a relevant dimension in the design space. Energy was taken for granted when ICT solutions were designed and then evolved over the years.

If ICT consumption is staggering today, the forecast of its growth is even more worrisome; in [4], for example, it is estimated that the carbon emission of the ICT sector could reach $14 \%$ of the total by 2040 . This is due to the expected increase in the pervasiveness of ICT. The well-known Cisco predictions [5] offer the trend of growth in internet traffic that is reported in Figure 1. The data is given in exabyte $\left(10^{\wedge} 18\right.$ byte) per month, a huge number that triples in only 5 years.

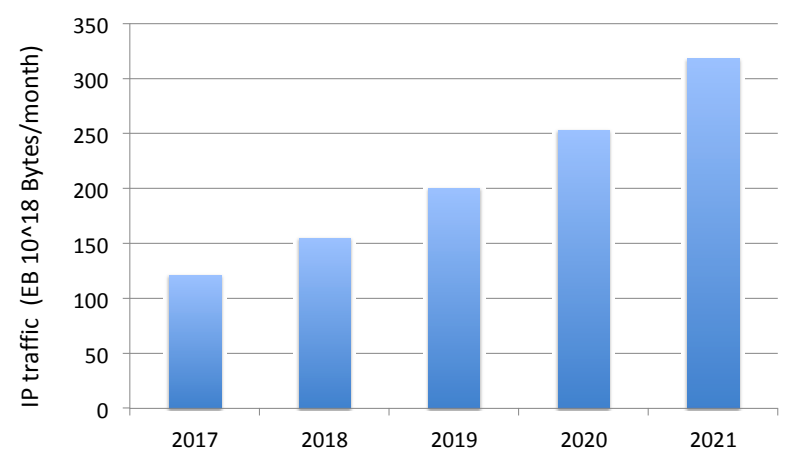

Fig. 1 Internet traffic predictions.

There are several reasons for this growth. The first is the popularity of high bit rate services that are based on multimedia streams, such as internet television and video calls. Figure 2, also based on Cisco predictions, shows the traffic growth for several services, and the importance of video services is evident.

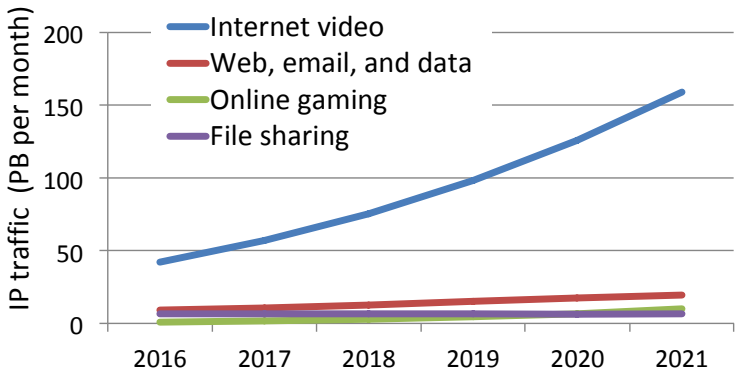

Fig. 2 Internet traffic per service.

Secondly, the number of users is growing worldwide, especially in developing areas: the compound annual growth rate of the number of internet users is $50 \%$ in the Middle-East and Africa. The number of connected devices, known as Internet of Things, from sensors, to machines and robots, is already estimated to be three times the world population, and, again, growth is expected to be extremely fast, corresponding to a growth factor 5 in 10 years, as can be seen from Figure 3, which is based on the data and predictions of [6].

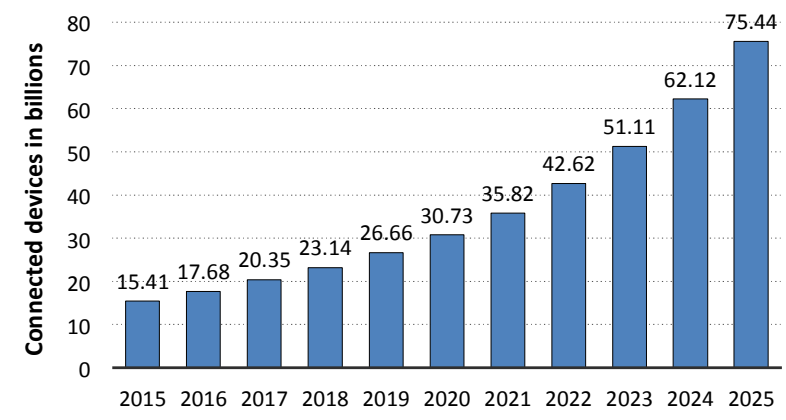

Fig. 3 Number of connected devices.

The combination of these factors pushes the deployment of enhanced communication infrastructure, the strengthening of communication and computing facilities, and terminals and devices, with a corresponding increase in electricity consumption and carbon emissions. The sustainability of the ICT sector is becoming an urgent need.

\section{Directions for action}

As previously discussed, the threat to ICT sustainability arises from the combination of the foreseen growth of communication and information services and the fact that the traditional design of ICT has not considered consumption and energy supply as a key issue, in all cases except when devices have to rely on small batteries. As a consequence, all aspects, from the device architecture to the protocols for communications, to resource management strategies and the several mechanisms hidden in a communication and information management infrastructure, have a significant margin of improvement for reducing their consumption. Beyond the enhancement of the electronics that improve the energy efficiency of the individual devices, there are 
three main directions that can be taken when considering the ICT infrastructure as a whole.

\subsection{Use of renewable energy}

Sustainability calls for new energy generation principles, that is, for the use renewable energy, however, its introduction in the communication and information infrastructure of renewable energy sources, such as solar panels and wind turbines, with their intermittent nature and variability in generation, is not straightforward at all. Indeed, it requires a re-design of architectures, and strategies to overcome the assumption that a power supply is continuous and guaranteed. We can consider a few examples. The scheduling of tasks and activities among a set of computing facilities, such as a set of servers, should aim not only at performance maximisation but also at consumption minimisation, and it should take into account the availability of energy, and the energy efficiency of the devices. Another example involves access networks that provide connectivity to users. The introduction of an intermittent power supply requires that the network is capable of self-reconfiguring so as to adapt to the possible unavailability of nodes due to energy shortage. Connectivity should also be provided only if necessary and in proportion to what is actually needed, so as to reduce energy waste.

In addition to sustainability, power supply through renewable sources is also advantageous in two types of scenarios: emerging countries and emergency situations. In emerging countries, the demand for communication services typically grows faster than the development of the power distribution infrastructure; networks and information infrastructure thus operate under an unreliable and discontinuous power supply. To guarantee continuity of operation, the network elements are often powered with costly and polluting diesel generators. This is the case for the base stations of cellular networks. In [7], it is estimated that 1 million base stations already operate without counting on a reliable power grid, and one third of these base stations are completely off-grid. In these scenarios, renewable energy sources are costeffective alternatives. In emerging situations, communications becomes fundamental but, frequently, also unavailable because of the unavailability of the power grid. In these cases, networks that can rely on alternative sources and can self-sustain are needed.

\subsection{Policies and rules}

There are a number of actions in terms of policy making and regulations that may potentially be quite effective. These actions aim at inducing more sustainable attitudes and adopting technologies that are more parsimonious in terms of resource usage. The ICT world experiences such fast evolution that policies and rules often do not keep pace; sustainability, however, requires urgent intervention and effective actions.

The use of electronic devices, and in particular terminals such as laptops and smartphones, involves an extremely fast turn-over that is not justified by the technological evolution but is rather pushed by an extremely profitable market. At the same time, device end-of-life is not managed and controlled as it should be, with harmful effects for the environment. Regulations about device end of life and the promotion of more careful attitudes by users, are both needed.

The presence of competing companies running communication infrastructure induces a waste of resources. The phenomenon is particularly evident at the periphery of the networks. Here, while service demand is variable according to the typical day/night patterns that follow human behaviour, the networks are continuously running at full capacity and consuming almost the same amount of energy as if they were working at full load. This inefficiency is multiplied by the number of overlapping networks corresponding to different technologies and different operators. Incentives for infrastructure sharing, a sort of cross-technology and cross-operation optimisation of the use of the infrastructure, would be very beneficial to reduce these inefficiencies.

Undesired and unnecessary information delivery, in the form of uncontrolled advertisements and spamming, comprises a considerable fraction of the total amount of information that is daily carried by the networks. Rules and mechanisms to control this might reduce this unnecessary and generally unwanted, but costly, information delivery.

\subsection{Users in the loop}

Users can effectively contribute to making ICT more sustainable by adopting proper behaviours as consumers of communication services, but people first need to be aware that there are sustainability issues around ICT and that, contrary to our perceptions, communication services consume huge amounts of resources. The first preliminary action involves disseminating knowledge about ICT sustainability and promote energy-aware attitudes.

Users can improve sustainability through a number of behaviours. First, they can choose less demanding services whenever possible. There are often several alternative services, of the same kind of quality, but different in terms of consumption, due to the amount of information that has to be carried and the distance in the internet that the information has to cover. Making choices with an awareness of these differences, if practiced by a large number of users, can have a significant aggregated impact.

Conscious choices by a large number of consumers might offer a competitive advantage on the market to solutions that are parsimonious in the use of resources, sparking a virtuous circle in which service providers design more sustainable solutions.

Crowdsourcing projects could also be started to monitor resource waste, collect data and warnings about possible critical situations, share suggestions on good practices, and so on. 


\section{References}

1. Ad-hoc Advisory Group "ICT for Energy Efficiency" of the European Commission DG INFSO, 2008.

2. H. Rong, H. Zhang, S. Xiao, C. Li, C. Hu, Optimizing energy consumption for data centers, Elsevier Renewable and Sustainable Energy Review, 58, 674-61 (2016).

3. R. Danilak, Why Energy Is A Big And Rapidly Growing Problem For Data Centerss, Forbes, 15/12/17.

4. L. Belkhir, A. Elmeligi, Assessing ICT global emissions footprint: Trends to 2040 \& recommendations, Elsevier Journal of Cleaner Production, 177, 448-463 (2018).

5. Cisco Visual Networking Index (VNI), 2017 [Online].

Available: https://www.cisco.com/c/en/us/solutions/collateral/s ervice-provider/visual-networking-index-vni/whitepaper-c11-741490.html [Access date: 15th, March 2019].

6. Statista [Online]. Available: www.statista.com [Access date: 15th, March 2019].

7. Green Power Design Approach and Feasibility Analysis," Green power for Mobile Technical White Paper, Aug. 2014. 